\title{
Analysis of Microindentation Unloading Curves based on Representative Strain Approach with Closed-Form Applications*
}

\author{
Nagahisa OGASAWARA**, Norimasa CHIBA** and Xi CHEN*** \\ ${ }^{* *}$ Department Mechanical Engineering, National Defense Academy \\ 1-10-20 Hashirimizu, Yokosuka, Kanagawa 239-8686, Japan \\ E-mail: oga@nda.ac.jp \\ *** Department of Civil Engineering and Engineering Mechanics, Columbia University \\ 628 S.W.Mudd Building, 500 W. 120 St., New York, NY 10027-6699, USA
}

\begin{abstract}
Indentation analysis based on the representative strain offers an effective way of obtaining material elastoplastic properties from the reverse analysis of indentation load-displacement curve. In this paper, its approach is employed to analyze the unloading force-depth curves obtained from a sharp microindentation test. By using the unloading work and residual penetration depth as parameters characterizing unloading, two different formulations of representative strain/stress are proposed, respectively, with very simple functional forms. When combined with the established framework of loading curves, the plastic properties and/or elastic properties of a material can be derived in closed-form using the loading curvature, unloading work, and residual depth measured from one sharp indentation test.
\end{abstract}

Key words: Indentation, Plastic Property, Elastic Property, Representative Strain, Dimensionless Function, Power-Law Material, Finite Element Method

\section{Introduction}

Microindentation is arguably one of the quickest and simplest ways of measuring the mechanical properties of materials, which involves very little effort on sample preparation, thus suitable for small material structures ${ }^{(1,2)}$. During the experiment, a sharp rigid indenter penetrates normally into a homogeneous solid where the indentation load, $P$, and displacement, $\delta$, are continuously recorded during a complete loading-unloading cycle (Fig. 1). To simplify the analysis, the indenter is usually modeled as a rigid cone with half apex angle $\alpha=70.3^{\circ}$, such that the ratio of cross-sectional area to depth is the same as that for a Berkovich or Vickers indenter with almost the same indentation response $(P-\delta$ curve) ${ }^{(3)}$. Denote the Young's modulus by $E$ and yield stress by $\sigma_{y}$, without losing generality, for an isotropic stress-free bulk material, its uniaxial stress-strain $(\sigma-\varepsilon)$ curve can be expressed in a power-law form (Fig. 2):

$$
\sigma=E \varepsilon \text { for } \varepsilon \leq \sigma_{y} / E \text { and } \sigma=R \varepsilon^{n} \text { for } \varepsilon \geq \sigma_{y} / E,
$$

where $n$ is the work-hardening exponent and $R \equiv \sigma_{y}\left(E / \sigma_{y}\right)^{n}$ is the work-hardening rate. When $n$ is zero, Eq. (1) reduces to an elastic-perfectly plastic material. For most metals and alloys $n$ is between 0.1 and 0.5 .

Upon indentation, if the yield strain $\sigma_{y} / E$ is relatively small (especially for the ductile metals/alloys that are most suitable for indentation test as well as our main focus in this paper), the material develops a pile-up due to the plastic flow of finite plastic 
deformation (Fig. 1(a)), which makes it difficult to accurately measure the projected contact area $A=\pi a^{2}$ at the maximum penetration ${ }^{(3)}$. It is therefore desirable to deduce material properties solely from the load-displacement curve where both $P$ and $\delta$ can be measured fairly accurately.

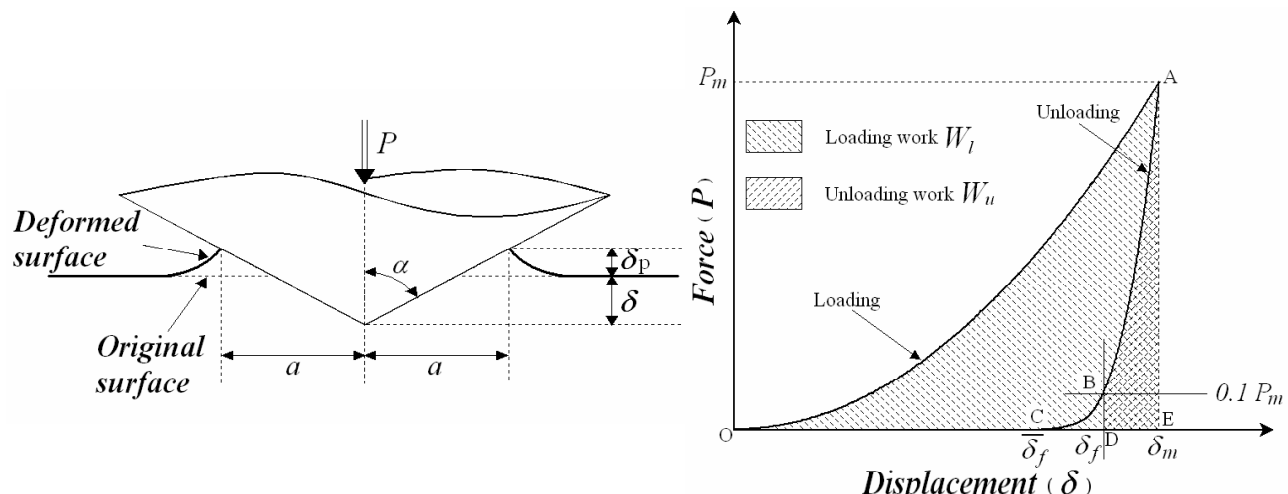

Fig. 1 Schematics of (a) a sharp indentation test with conical indenter (whose half-apex angle is $\alpha$ ); (b) typical indentation force-depth $(P-\delta)$ curve measured from an indentation experiment.



Fig. 2 Schematic of uniaxial stress-strain $(\sigma-\varepsilon)$ curve of materials obeying the power-law hardening law (1). Also shown is the definition of representative strain $\varepsilon_{R}$ and representative stress $\sigma_{R}$.

When the indenter apex angle is fixed and the minor effects from the Poisson's ratio is neglected, based on dimensional analysis ${ }^{(4)}$ the $P-\delta$ data measured during loading can be related with material elastoplastic properties:

$$
C \equiv P / \delta^{2}=\sigma_{y} \cdot \Psi\left(\sigma_{y} / E, n\right)
$$

$C$ is also referred to as the loading curvature. Owing to the self-similarities of the dimensionless function $\Psi$, a mathematical trick known as the representative strain may be introduced $^{(5)}$ to reduce the number of apparent unknown variables in $\Psi$ down to one. In essence, for a given indenter apex angle, there exists a representative strain $\varepsilon_{R}$ (which is only a function of $\alpha$ ) and corresponding representative stress $\sigma_{R}$ (whose formulation depends on $n$ and plastic properties of a specific material), such that the new dimensionless function

$$
\frac{C}{\sigma_{R}}=\Xi\left(\frac{\bar{E}}{\sigma_{R}}\right)
$$

depends only on $\bar{E} / \sigma_{R}$, where $\bar{E}=E /\left(1-v^{2}\right)$ is the plane strain modulus. Both representative strain $\varepsilon_{R}$ and $\Xi$ can be determined from fitting of extensive numerical analyses; for a given $\alpha$, there are various definitions (formulations) of the representative stress and strain ${ }^{(3,5-15)}$. If the modulus of the material $(\bar{E})$ is known a priori, the only unknown variable $\sigma_{R}$ can then be solved from experimental $P-\delta$ data along with (3); such solution procedure is often numerical if the functional form of $\Xi$ is complex (e.g. 
polynomial or power-law ${ }^{(5-13)}$ ).

However, the knowledge of $\sigma_{R}$ alone is insufficient in order to solve for the material plastic property, which involves two independent unknowns, $\sigma_{v}$ and $n$ (or equivalently, $R$ and $n$ ). In general, one could obtain an independent $P-\delta$ curve from another test with a different indenter angle (with a different set of $\varepsilon_{R}$ and $\Xi$ ), and various algorithms have been proposed for measuring the plastic properties using the dual indenter method ${ }^{(6-10,13-18)}$.

Although the dual indenter method can be used to determine the plastic properties accurately, it has several disadvantages: (1) It is sometimes not practical to obtain two indenters with distinct apex angles. Moreover, the indenter angles have to be the same as those proposed in the previous studies ${ }^{(6-10,13-16)}$; otherwise, one has to recalculate $\varepsilon_{R}$ and $\Xi$ for that specific $\alpha$; (2) The impression must be made twice on the same specimen and separated with a practical distance, which makes it impossible to obtain accurate information from one specific interested material point; (3) The rich information from the unloading curve was not used.

In this paper, we extend the convenient representative strain approach to unloading curves. By using either the unloading work or the residual penetration as the governing parameter, we propose two different formulations of representative strain and stress. Unlike conventional approaches where lengthy and complex polynomial and/or power-law fitting were used ${ }^{(5-13,16)}$, the formulations derived from this paper are very simple, involving at most two fitting parameters. When combined with the representative strain formulation for loading curves we established earlier based on limit analysis ${ }^{(14,15)}$ (which was also concise without fitting parameters), the concise functional form allows the material plastic and/or elastic properties to be derived analytically in closed-form. In other words, by simply measuring the loading curvature, unloading work, and residual penetration from one sharp indentation test (the Berkovich tip is used in this study), one can directly derive the material elastoplastic properties of power-law materials from simple closed-form formulae.

\section{Computation Method}

A typical load-depth $(P-\delta)$ curve is given in Fig. 1(b), where $P_{m}, \delta_{m}$ and $\bar{\delta}_{f}$ are the maximum indentation load, maximum indentation depth, and final residual penetration (after complete unloading), respectively. The work done by the indenter during loading is the area under the loading curve OA, i.e. $W_{l}=\int_{0}^{\delta_{m}} P d \delta=\int_{0}^{\delta_{m}} C \delta^{2} d \delta=\frac{1}{3} C \delta_{m}{ }^{3}$. Similarly, the final unloading work is the area beneath the unloading curve AC, i.e. $\bar{W}_{u}=-\int_{\delta_{u}}^{\bar{\delta}_{f}} P d \delta$. Note that the final residual penetration is sometimes difficult to be identified precisely because the unloading curve often has a long "tail" with almost zero slope (BC) when the indentation load approaches to zero. In order to minimize the error of measuring the residual penetration (in both practical and numerical experiments), a reduced residual penetration $\delta_{f}$ is defined as the penetration at $10 \%$ maximum indentation load (Fig. 1(b)). Correspondingly, the reduced unloading work becomes $W_{u}=-\int_{\delta_{m}}^{\delta_{t}} P d \delta$ (area of ABDE). For convenience, in the following text we will drop the "reduced" term and refer $\delta_{f}$ as the residual penetration (or residual depth) and $W_{u}$ as the unloading work.

Therefore, the primary shape factors characterizing a sharp indentation $P-\delta$ curve are: the loading curvature, $C \equiv P / \delta^{2}$, the unloading work, $W_{u}$, and the residual depth, $\delta_{f}$. The first one characterizes the loading behavior and the next two are for unloading. The relationships between these key variables of indentation are related with material elastoplastic properties $\left(E, \sigma_{v}, n\right)$, are established via numerical indentation tests using finite element method (FEM), where the material properties are varied in a large range such that the established functional forms can be applied to a large material space. 
Numerical calculations are performed using the commercial code ANSYS ${ }^{(19)}$. Selected results are verified by using another commercial code ABAQUS ${ }^{(20)}$. The rigid contact surface is used to simulate the rigid indenter, and the option for finite deformation and strain is employed. A typical mesh for the axisymmetric indentation model comprises about 10,000 4-node elements with reduced integration. Figure 3 shows the finite element model. The minimum element size is $15 \mathrm{~nm}$ x $25 \mathrm{~nm}$. To avoid the boundary effect, the penetration depth is less than $1 \%$ of the model size which is $100 \mathrm{~nm} \times 200 \mathrm{~nm}$. This model is considered as semi-infinite body. The material parameters are varied over a large range to cover essentially all engineering materials, with $\bar{E} / \sigma_{R}=3 \sim 4000$ and $n=0 \sim 0.5^{(15)}$. A total of 71 different combinations of material properties are examined (with $n=0.0 \sim 0.5$ ). Note that for larger $n$ values, the range of $\bar{E} / \sigma_{y}$ is much larger than $\bar{E} / \sigma_{R}$. The Coulomb's friction law is used between contact surfaces, and the friction coefficient is taken to be 0.15 (21), which is a minor factor for indentation as long as this value is relatively small. This has been verified by our FEM analyses. Bucaille et al. ${ }^{(7)}$ have carried out extensive studies on the friction effect for the conical indentation and found similar results. The Poisson's ratio is fixed at 0.3 for all materials, which is consistent with the literature.

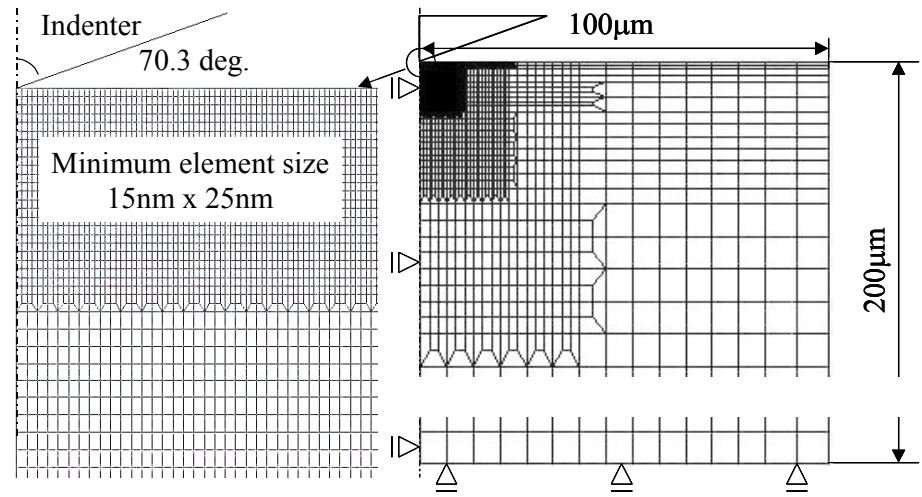

Fig. 3 Finite element mesh used in this research.

\section{Representative Strain Approach for Unloading Curve}

\subsection{Definition of the Representative Strain during Loading}

For loading $P-\delta$ curves, many researchers ${ }^{(7-11,16-18)}$ have followed the approach of Dao et al. (5) to define $\varepsilon-\sigma_{y} / E$ as the representative strain (where $\varepsilon$ is the uniaxial strain, (1)), which was found to be 0.033 for Berkovich indenter. This definition has weak physical basis and its working range is relatively small ${ }^{(6)}$. Inspired by the fact that conical indentation is axisymmetric, we have proposed to define $\varepsilon_{R}$ as the plastic strain during axisymmetric deformation ${ }^{(6)}$, and the corresponding representative stress is (see the uniaxial stress-strain curve of a power-law material in Fig. 2):

$$
\sigma_{R}=R\left(2 \sigma_{R} / E+2 \varepsilon_{R}\right)^{n} \text {. }
$$

This formulation not only has better physical background, but it also works with the best accuracy (compare with other possible formulations) in a wider range of material properties

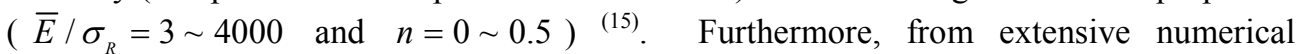
analyses, the representative strain is identified as a function of the apex angle of conical indenter: ${ }^{(6)}$

$$
\varepsilon_{R}=0.0319 \cot \alpha .
$$

In this study, we focus on the Berkovich indenter only, which gives $\varepsilon_{R}=0.0115$.

When the material is either elastic or rigid plastic, the indentation problem can be solved in closed form. Thus, based on our previous limit analysis ${ }^{(14)}$, the normalized loading curvature can be related with $\bar{E} / \sigma_{R}$ as: 


$$
\frac{C}{\sigma_{R}}=\frac{3 W_{l}}{\sigma_{R} \delta_{m}{ }^{3}}=\left(\frac{1}{m_{e}{ }^{l} \cdot \frac{\bar{E}}{\sigma_{R}}}+\frac{1}{m_{p}^{l}}\right)^{-1}
$$

The results are given in Fig. 4 where symbols are results obtained from FEM analyses and line is Eq. (6). For an elastic solid $\left(\bar{E} / \sigma_{R} \rightarrow 0\right), \frac{C}{\sigma_{R}}=\frac{2 \gamma}{\pi} \tan \alpha \cdot \frac{\bar{E}}{\sigma_{R}} \equiv m_{e}^{l} \cdot \frac{\bar{E}}{\sigma_{R}}$, with $m_{e}^{l}=\frac{2 \gamma}{\pi} \tan \alpha$ as the elastic limit for loading curve, where $\gamma$ is a correction factor proposed by Hay et al. ${ }^{(22)}$, i.e. $\gamma=\pi\left(\pi / 4+0.155 \cot \alpha \frac{1-2 v}{4(1-v)}\right) /\left(\pi / 2-0.831 \cot \alpha \frac{1-2 v}{4(1-v)}\right)^{2}$ for Berkovich tip; for a rigid plastic solid $\left(\bar{E} / \sigma_{R} \rightarrow \infty\right), C / \sigma_{R}$ approaches the plastic limit $m_{p}^{l}$ for loading. $m_{p}^{l}$ can be either derived from Mises yield condition, which equals to 114.7 for Berkovich tip (and thus there is no fitting parameter in Eq. (6)); alternatively, the limit $m_{p}^{l}$ can be easily fitted from FEM analysis (symbols in Fig. 4 as $\bar{E} / \sigma_{R}$ gets large) to ensure maximum accuracy, while involving only one fitting parameter $\left(m_{p}^{l}=112.1\right.$ for Berkovich indenter) that has a strong physical basis. Both elastic and plastic limits are embedded in the loading equation, Eq. (6), from which the representative stress can be obtained explicitly as $\sigma_{R}=\frac{m_{e}^{l} C \bar{E}}{m_{p}^{l}\left(m_{e}^{l} \bar{E}-C\right)}$ without any iteration.

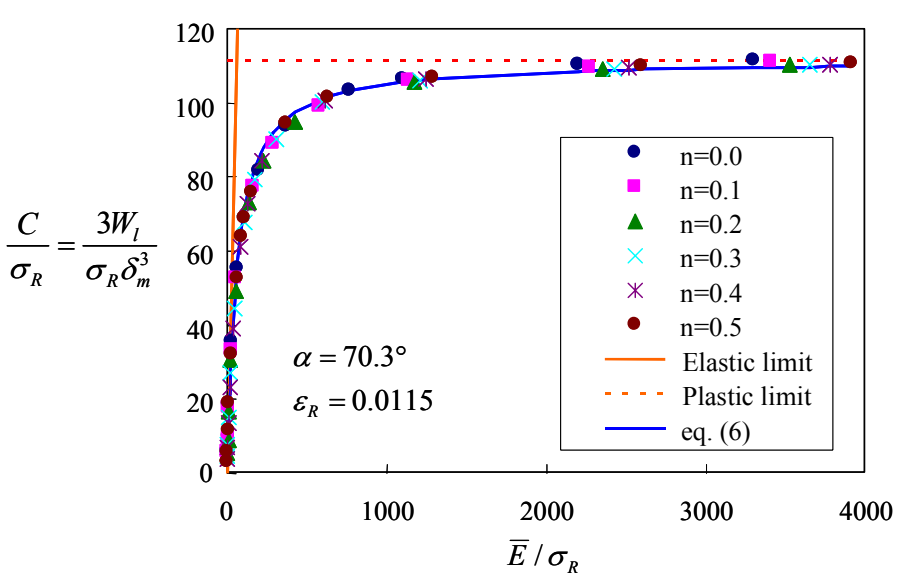

Fig. 4 Relationship between $C / \sigma_{R}$ and $\bar{E} / \sigma_{R}$ for Berkovich indentation on a wide range of materials, which is apparently independent of the work hardening exponent $\mathrm{n}$. Also shown are the elastic and plastic limits, and the limit-analysis-based equation (6).

\subsection{Extend the Representative Strain to Unloading}

We now extend the same representative strain and stress defined above (Fig. 2 and Eq. (4)) to unloading. By inspecting the unloading curve in Fig. 1(b), whose shape is similar to an triangle with the enclosed area almost proportional to $\left(\delta_{m}-\delta_{f}\right)$; thus, when the unloading work is normalized by $\delta_{m}^{2}\left(\delta_{m}-\delta_{f}\right)$ instead of $\delta_{m}^{3}$, it becomes apparently independent of $n$, which the relation is shown in Fig. 5; moreover, due to its similarity to Eq. (6), the behavior can be represented by a limit-analysis-based form: 


$$
\frac{W_{u}}{\sigma_{R} \delta_{m}^{2}\left(\delta_{m}-\delta_{j}\right)}=\left(\frac{1}{m_{e} \frac{\bar{E}}{\sigma_{R}}}+\frac{1}{m_{p}^{u}}\right)
$$

If the material is elastic ( $\left.\bar{E} / \sigma_{R} \rightarrow 0\right)$, from Fig. 1(b)

$$
\delta_{f}=\sqrt{0.1 \frac{P_{m}}{C}}=\sqrt{0.1} \cdot \delta_{m}
$$

Thus, the unloading work is

$$
W_{u}=-\int_{\delta_{m}}^{\delta_{f}} P d \delta=\frac{C}{3}\left(\delta_{m}{ }^{3}-\delta_{f}{ }^{3}\right)=\left(1-\sqrt{0.1}^{3}\right) W_{l}
$$

The normalized variable becomes

$$
\frac{W_{u}}{\sigma_{R} \delta_{m}{ }^{2}\left(\delta_{m}-\delta_{f}\right)}=\frac{W_{l}\left(1-\sqrt{0.1}^{3}\right)}{\sigma_{R} \delta_{m}{ }^{3}(1-\sqrt{0.1})}=1.416 \frac{W_{l}}{\sigma_{R} \delta_{m}{ }^{3}}
$$

The representative stress of an elastic material is infinity, which leads to

$$
W_{l}=\frac{\delta_{m}{ }^{3}}{1.416} m_{e}^{u} \bar{E}
$$

And from Eq. (6), for an elastic material

$$
W_{l}=\frac{\delta_{m}{ }^{3}}{3} m_{e}^{l} \bar{E}
$$

Equating (11) and (12) leads to

$$
m_{e}^{u}=\frac{1.416}{3} m_{e}^{l}=0.887
$$

Meanwhile, if the material is very plastic $\left(\bar{E} / \sigma_{R}\right.$ very large), $W_{u}$ is very small and the unloading $P-\delta$ curve is almost straight, which can be approximated by

$$
W_{u}=\frac{1}{2}\left(\delta_{m}-\delta_{f}\right) \cdot 0.9 P_{m}+\left(\delta_{m}-\delta_{f}\right) \cdot 0.1 P_{m}=0.55\left(\delta_{m}-\delta_{f}\right) \cdot C \delta_{m}^{2}
$$

Considering that the representative stresses are same for both loading and unloading in this section, and that $\bar{E} / \sigma_{R}$ is very large,

$$
\frac{W_{u}}{\delta_{m}{ }^{2} \cdot\left(\delta_{m}-\delta_{f}\right) \cdot \sigma_{R}}=0.55 \frac{C}{\sigma_{R}}
$$

and thus

$$
m_{p}^{u}=0.55 m_{p}^{l}=63.1
$$

based on Mises yield criterion; in this case, there is no fitting parameter involved in the formulation. Alternatively, it is also fairly easy to fit the plastic limit $m_{p}^{u}$ from the FEM data points shown in Fig. 5, which leads to $m_{p}^{u}=59.0$ and with only one fitting parameter, the analysis may obtain better accuracy. The functional form of Eq. (7) is plotted as a line in Fig. 5, which shows that such simple formulation based on residual depth works fairly well. 


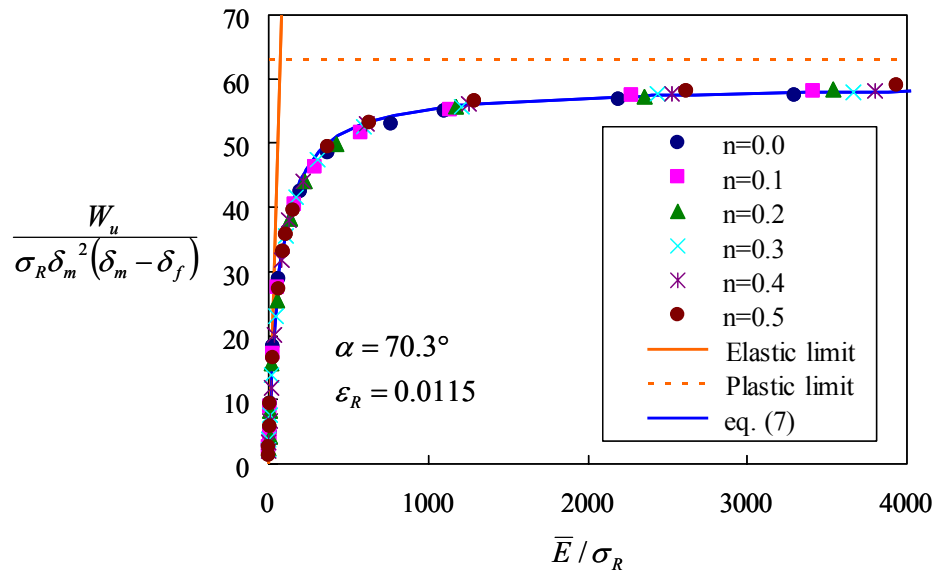

Fig. 5 Relationship between $\frac{W_{u}}{\sigma_{R} \delta_{m}^{2}\left(\delta_{m}-\delta_{f}\right)}$ and $\bar{E} / \sigma_{R}$ for Berkovich indentation on a wide range of materials, which is apparently independent of the work hardening exponent $n$. Also shown are the elastic and plastic limits, and the limit-analysis-based equation (7).

\subsection{A Different Representative Stress based on Unloading Work}

We retain the same value of the representative strain, Eq. (5), and seek for a new representative stress for unloading, $\sigma_{r^{\prime}}$, such that the normalized unloading work is related with both $\bar{E}$ and $C$, but is essentially independent of $n$ for the same range of materials described above. The representative stress $\sigma_{r}$ is given by

$$
\sigma_{r^{\prime}}=R\left(1.47 \cdot \frac{\sigma_{r^{\prime}}}{E}+3.22 \cdot \varepsilon_{R}\right)^{n}
$$

with $R=\sigma_{y}\left(E / \sigma_{y}\right)^{n}$. This is a robust formulation for $\alpha$ between $60^{\circ}$ and $80^{\circ}$; for Berkovich indenter, the results of the normalized unloading work are given in Fig. 6(a), which can be fitted by the following equation

$$
\frac{\delta_{m}^{3} \cdot \sigma_{r^{\prime}}}{W_{u}}=a_{3} \cdot \frac{\bar{E}}{\sigma_{r^{\prime}}}+b_{3} \frac{\sigma_{r^{\prime}}}{C}+c_{3}
$$

for a wide range of elastoplastic materials, with $a_{3}=0.00118, b_{3}=2.47, c_{3}=0.0830$ for the Berkovich tip (these numbers depend on $\alpha$ ). If both $W_{u}$ and $C$ are measured and the material modulus is known, the representative stress is (which is different from $\sigma_{R}$ discussed above although $\varepsilon_{R}$ keeps the same):

$$
\sigma_{r^{\prime}}=\frac{c_{3}+\sqrt{c_{3}{ }^{2}+4\left(\frac{\delta_{m}{ }^{3}}{W_{u}}-\frac{b_{3}}{C}\right) a_{3} \bar{E}}}{2\left(\frac{\delta_{m}{ }^{3}}{W_{u}}-\frac{b_{3}}{C}\right)}
$$

To further simplify the formulation, we note that the relationship in Fig. 6(a) becomes monotonic when $\bar{E} / \sigma_{r}$, is larger than about 25 , which includes most engineering metals and alloys (since $\sigma_{r}>\sigma_{y}$ ), in particular the ductile materials of our focus in this paper. If only these more plastic materials are of concern, the effect of $C$ becomes less important and another unloading representative stress $\sigma_{r}$ can be derived, such that the relationship between $\sigma_{r} \delta_{m}{ }^{3} / W_{u}$ and $\bar{E} / \sigma_{r}$ is almost linear and independent of $n$, which can be fitted by

$$
\frac{\delta_{m}^{3} \sigma_{r}}{W_{u}}=a_{2} \frac{\bar{E}}{\sigma_{r}}+b_{2} .
$$

In case $\alpha=70.3^{\circ}, a_{2}=0.000951$ and $b_{2}=0.139$, and the results are shown in Fig. 
6(b). For other $\alpha$ values, the coefficients $a_{2}$ and $b_{2}$ are different. The new $\sigma_{r}$ that could best eliminate the $n$-dependence in (20) is

$$
\sigma_{r}=R\left(1.3 \frac{\sigma_{r}}{E}+2.6 \varepsilon_{R}\right)^{n},
$$

which is valid for $\alpha$ between $60^{\circ}$ and $80^{\circ}$. While Eqs. (18) and (17) are valid for a large material space, Eqs. (20) and (21) are recommended for the more plastic materials with $\bar{E} / \sigma_{r}>30$ or so. Next, we apply the representative stresses derived in these sections, in particular those new formulations proposed for unloading behavior, to obtain material elastoplastic properties in closed-form.
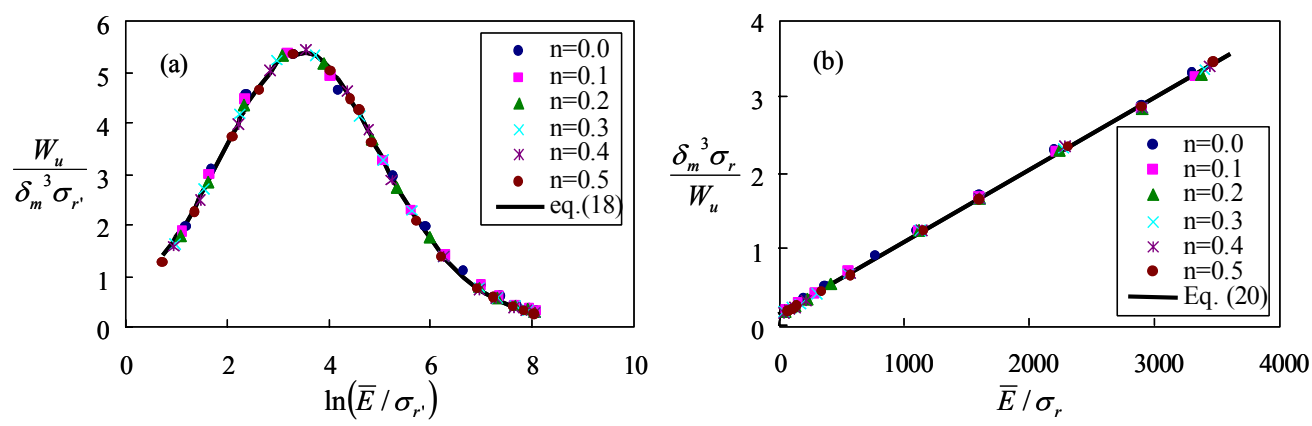

Fig. 6 Relationship between the normalized unloading work and normalized modulus: (a) for a wide range of materials, where $\sigma_{r}$, is used as the representative stress and eq. (18) is employed; (b) for the more

plastic materials, where $\sigma_{r}$ is used as the representative stress and eq. (20) is employed.

\section{Obtaining Material Properties via Closed-form Formulae}

\subsection{Derive Plastic Properties of Ductile Materials}

We first develop a closed-form algorithm to determine the plastic properties of the specimen from the load-displacement curve of just one indentation test. Note that once the material composition is known, its elastic modulus can be estimated in many cases. For example, while alloying and/or processing can significantly alter the material plastic behavior, they can hardly change the elastic modulus. Thus, we focus on the plastic property measurement in this section. From an indentation experiment, the loading curvature $C=P / \delta^{2}$ can be obtained from the average of loading curve; alternatively, one can compute the loading work $W_{l}$ by integrating the loading $P-\delta$ curve and then derive $C=3 W_{l} / \delta_{m}{ }^{3}$. The unloading work $W_{u}$ can be obtained from that described in Section 2 .

With $\frac{m}{E}$ (and E) known, according to Eqs. (4) and (6), one flow stress-total strain point can be determined on the uniaxial stress-strain curve as

$$
\sigma_{1}^{t}=\frac{m_{e}^{l} C \bar{E}}{m_{p}^{l}\left(m_{e}^{l} \bar{E}-C\right)} \quad \text { and } \quad \varepsilon_{1}^{t}=2 \sigma_{1}^{t} / E+2 \varepsilon_{R}
$$

Next, based on Eqs. (20) and (21), from the unloading work measurement another flow stress-total strain point can be derived

$$
\sigma_{2}^{t}=\frac{b_{2}+\sqrt{b_{2}{ }^{2}+4 a_{2} \bar{E} \delta_{m}{ }^{3} / W_{u}}}{2 \delta_{m}{ }^{3} / W_{u}} \quad \text { and } \quad \varepsilon_{2}^{t}=1.3 \frac{\sigma_{2}^{t}}{E}+2.6 \varepsilon_{R}
$$

Finally, the material plastic parameters can be derived in closed form:

$$
n=\frac{\ln \left(\sigma_{1}^{t} / \sigma_{2}^{t}\right)}{\ln \left(\varepsilon_{1}^{t} / \varepsilon_{2}^{t}\right)} \quad, \quad R=\frac{\sigma_{1}^{t}}{\left(\varepsilon_{1}^{t}\right)^{n}} \quad, \quad \sigma_{y}=\left(\frac{E^{n}}{R}\right)^{\frac{1}{n-1}}
$$

Eqs. (22)-(24) represent a new closed-form technique for deriving the material plastic properties via Berkovich indentation test, and it is recommended for the more plastic 
materials. Note that one could also use Eqs. (17) and (19) instead of (20) and (21) in the above step, and the formulation will be more suitable for the more elastic materials.

\subsection{Derive Elastic and Plastic Properties of Metals}

If the Young's modulus of the material is unknown, from Eqs. (6) and (7), one can derive the plane strain modulus and a flow stress (representative stress) in closed form, once $W_{l}, W_{u}, \delta_{m}$, and $\delta_{f}$ are measured from an indentation test:

$$
\begin{aligned}
& \bar{E}=\frac{3\left(m_{e}^{u} m_{p}^{l}-m_{e}^{l} m_{p}^{u}\right) W_{l} W_{u}}{\delta_{m}{ }^{2} m_{e}{ }_{e} m_{e}^{u}\left(\delta_{m} m_{p}^{l} W_{u}-3 m_{p}^{u} W_{l}\left(\delta_{m}-\delta_{f}\right)\right)} \\
& \sigma_{1}^{t}=\sigma_{R}=\frac{3\left(m_{e}^{u} m_{p}^{l}-m_{e}^{l} m_{p}^{u}\right) W_{l} W_{u}}{\delta_{m}{ }^{2} m_{p}^{l} m_{p}^{u}\left(-\delta_{m} m_{e}^{l} W_{u}+3 m_{e}^{u} W_{l}\left(\delta_{m}-\delta_{f}\right)\right)}
\end{aligned}
$$

The corresponding total strain is $\varepsilon_{1}^{t}=2 \sigma_{1}^{t} / E+2 \varepsilon_{R}$. Similar to Section 4.1, using Eqs. (20), (21), and (25), another flow stress-total strain point can be derived:

$$
\sigma_{2}^{t}=\frac{b_{2}+\sqrt{b_{2}{ }^{2}+4 a_{2} \frac{3 \delta_{m}\left(m_{e}^{u} m_{p}^{l}-m_{e}^{l} m_{p}^{u}\right) W_{l}}{m_{e}^{l} m_{e}^{u}\left(\delta_{m} m_{p}^{l} W_{u}-3 m_{p}^{u} W_{l}\left(\delta_{m}-\delta_{f}\right)\right)}}}{2 \delta_{m}^{3} / W_{u}} \text { and } \varepsilon_{2}^{t}=1.3 \frac{\sigma_{2}^{t}}{E}+2.6 \varepsilon_{R}
$$

Finally, material parameters $\left(E, \sigma_{y}, n\right)$ can be derived in closed form using (25) and (24), which represent another closed-form technique for measuring material elastoplastic properties via a Berkovich indentation test.

\subsection{Applications of the Proposed Methods}

The proposed method is now applied to determine the properties of four ductile materials, whose power-law constitutive constants $(E, R, n)$ are tabulated in Table 1 and then used as the input data for the numerical indentation experiment with FEM. The thin lines in Fig. 7 show the input uniaxial stress-strain relationships.

First, we assume that the material Young's modulus $E$ is known a priori. Following Eqs. (22)-(24), the results obtained from the reverse analysis for 4 materials are shown in Fig. 7 as thick solid lines for the identified stress-strain relationships; the numerical results are also tabulated in Table 1. In all cases excellent agreement is found between the extracted plastic properties and input data, validating the robustness of the proposed method for ductile materials. We caution that such method for determining plastic properties is relatively sensitive to the error of $E$. When $E$ is perturbed by $1 \%$, additional error of about $5-10 \%$ may appear for the determined plastic properties. This is mainly because the indentation $P-\delta$ curve is more dependent on the elastic modulus than the plastic properties, and thus the formulation based on the $P-\delta$ curve as proposed in this paper is relatively sensitive to the variation of $E$.

To further confirm the effectiveness of the improved technique, we study several materials that have been previously claimed as not being able to be characterized by single indentation test. Alkorta et al. ${ }^{(23)}$ have shown that there are combinations of materials that have identical load-displacement curves, and the examples of such materials are given in Table 1 of ref. (23); from Fig. 8(a) we can see that indeed, the indentation load-depth curves of these materials are indistinguishable. Nevertheless, by applying the proposed formulation, we are able to identify the plastic properties of all five materials with satisfied accuracy: In Fig. 8(b), the thin lines are input data (according to Table 1 of Alkorta et al. ${ }^{(23)}$ ) and the thick lines are results obtained from our reverse analysis. The good agreement shows that if the elastic modulus is known, the proposed closed-form method is very useful and easy to determine the material plastic properties by just one indentation test. 


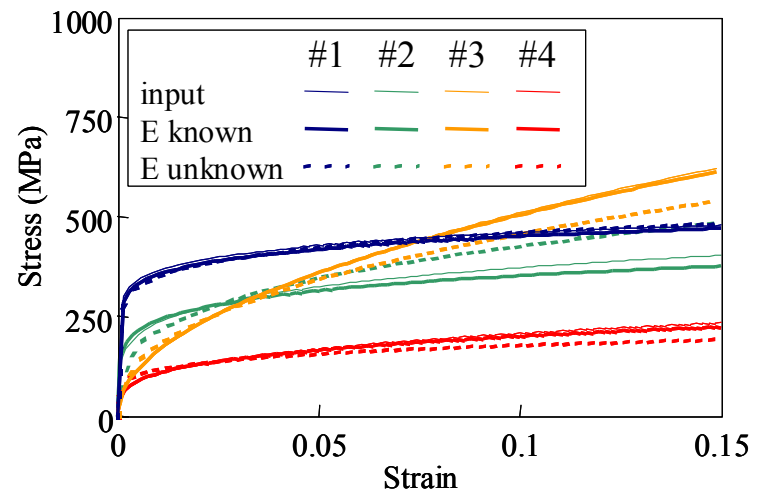

Fig. 7 Comparisons between the uniaxial stress-strain curves based on input properties in Table 1 (thin lines), and reverse analysis results from closed-form formulae (thick solid lines based on method in Section

4.1 with $E$ known, and thick dash lines based on method in Section 4.2 with $E$ unknown).

Table 1 Comparisons between the input material properties and those obtained from the reverse analysis of proposed formulae.

\begin{tabular}{|c|l|c|c|c|c|c|}
\hline & & input & Sec. 4.1 & error (\%) & Sec. 4.2 & error (\%) \\
\hline \multirow{3}{*}{$\# 1$} & $\mathrm{E}(\mathrm{GPa})$ & 210 & known & - & 211 & 0.51 \\
\cline { 2 - 7 } & $\mathrm{n}$ & 0.10 & 0.10 & 4.08 & 0.12 & 18.6 \\
\cline { 2 - 7 } & $\mathrm{R}(\mathrm{MPa})$ & 578 & 572 & -0.95 & 603 & 4.36 \\
\hline \multirow{3}{*}{$\# 2$} & $\mathrm{E}(\mathrm{GPa})$ & 300 & known & - & 320 & 6.50 \\
\cline { 2 - 7 } & $\mathrm{n}$ & 0.20 & 0.16 & -18.2 & 0.30 & 52.0 \\
\cline { 2 - 7 } & $\mathrm{R}(\mathrm{MPa})$ & 589 & 512 & -13.1 & 858 & 45.8 \\
\hline \multirow{3}{*}{$\# 3$} & $\mathrm{E}(\mathrm{GPa})$ & 300 & known & - & 290 & -3.30 \\
\cline { 2 - 7 } & $\mathrm{n}$ & 0.50 & 0.49 & -2.21 & 0.42 & -16.6 \\
\cline { 2 - 7 } & $\mathrm{R}(\mathrm{MPa})$ & 1619 & 1563 & -3.45 & 1199 & -25.9 \\
\hline \multirow{3}{*}{$\# 4$} & $\mathrm{E}(\mathrm{GPa})$ & 300 & known & - & 287 & -4.19 \\
\cline { 2 - 7 } & $\mathrm{n}$ & 0.30 & 0.27 & -8.93 & 0.19 & -38.3 \\
\cline { 2 - 7 } & $\mathrm{R}(\mathrm{MPa})$ & 414 & 376 & -9.25 & 271 & -34.6 \\
\hline
\end{tabular}
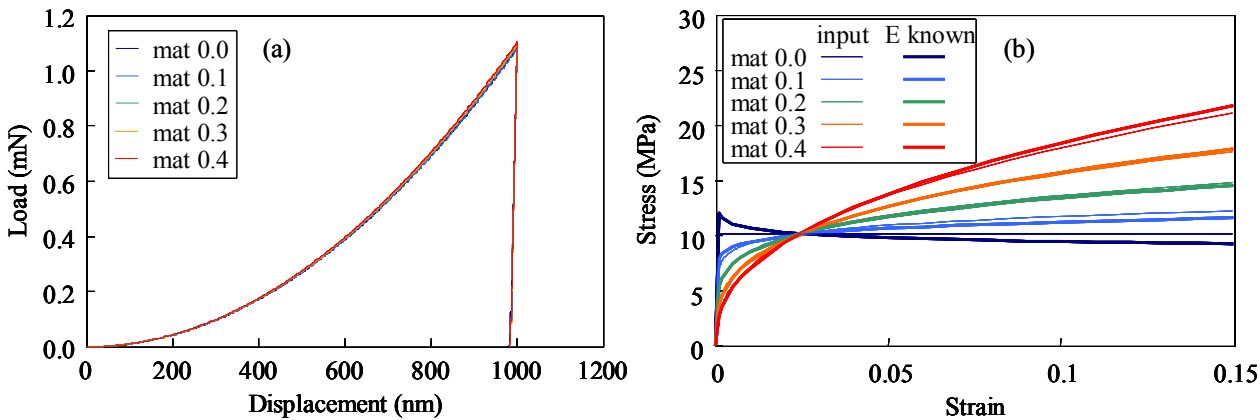

Fig. 8 For special materials presented in ref. (23), the plastic properties of these five materials can be accurately measured from the proposed reverse analysis with one indentation test.

Next, we take the material modulus as unknown, and following the closed-form solution in Section 4.2, the extracted elastoplastic properties (Table 1) lead to thick dash lines in Fig. 7: the comparison shows that when there are errors of the identified $E$, the errors of determined plastic properties increase as well. The error of the measured $E$ is relatively modest, however the errors of the determined plastic properties are enlarged by the perturbation of $E$, as discussed above. Nevertheless, the method still showed promising potentials for measuring the elastic and plastic properties of ductile materials from a sharp indentation test. In particular, the proposed functional forms, Eqs. (24)-(27) are simple and easy to use, which are perhaps the first set of closed-form formulae for deriving the material elastoplastic properties since the classic Oliver-Pharr approach ${ }^{(24)}$, yet unlike the Oliver-Pharr method, the approach in this paper is valid for power-law materials with strain hardening, and it does not require the measurement of the projected contact area. 


\section{Conclusion}

In summary, we propose closed-form formulae to determine the plastic or elastoplastic properties of a bulk material by using a sharp indentation test. We extend the representative strain approach from loading curve to unloading curve, and by using residual penetration and unloading work as variables, two formulations of representative strain and stress are proposed for the unloading force-depth curve of an indentation test. The functional forms are kept simple such that no fitting parameter may be involved based on limit analysis; at most 2 fitting parameters can be employed for pursuing the best accuracy. Demonstrated via examples of ductile materials, the reverse analysis results agree well with the input data used in numerical experiment, and it has been applied to correctly identify different material properties with almost identical load-displacement curves. Therefore, the closed-form method has the potential to easily and effectively measure the material by one indentation test.

\section{Acknowledgement}

The work is supported in part by NSF CMS-0407743 and NSF CMMI-CAREER-0643726, and in part by the Department of Civil Engineering and Engineering Mechanics, Columbia University.

\section{References}

(1) Chen, X. and Vlassak, J.J., Numerical study on the measurement of thin film mechanical properties by means of nanoindentation, Journal of Materials Research, 16 (2001) pp. 2974-2982.

(2) Zhao, M., Chen, X., Xiang, Y., Vlassak, J.J., Lee, D., Ogasawara, N., Chiba, N., and Gan, Y.X., Measuring elastoplastic properties of thin films on an elastic substrate using sharp indentation, Acta Materialia, 55 (2007) pp. 6260-6274.

(3) Chen, X., Ogasawara, N., Zhao, M., and Chiba, N., On the uniqueness of measuring elastoplastic properties from indentation: the indistinguishable mystical materials, Journal of the Mechanics and Physics of Solids, 55 (2007) pp. 1618-1660.

(4) Cheng, Y.T. and Cheng, C.M., Scaling approach to conical indentation in elastic-plastic solids with work hardening, J. Appl. Phys., 84 (1998) pp. 1284-1291.

(5) Dao, M., Chollacoop, N., VanVliet, K.J., Venkatesh, T.A., and Suresh, S., Computational modeling of the forward and reverse problems in instrumented sharp indentation, Acta Mater., 49 (2001) pp. 3899-3918.

(6) Ogasawara, N., Chiba, N., and Chen, X., Representative strain of indentation analysis, Journal of Materials Research, 20 (2005) pp. 2225-2234.

(7) Bucaille, J.L., Stauss, S., Felder, E., and Michler, J., Determination of plastic properties of metals by instrumented indentation using differnt sharp indenters, Acta Mater., 51 (2003) pp. 1663-1678.

(8) Chollacoop, N., Dao, M., and Suresh, S., Depth-sensing instrumented indentation with dual sharp indenters, Acta Materialia, 51 (2003) pp. 3713-3729.

(9) Swaddiwudhipong, S., Tho, K.K., Liu, Z.S., and Zeng, K., Material characterization based on dual indenters, Int. J. Solids Struct., 42 (2004) pp. 69-83.

(10) Wang, L., Ganor, M., and Rokhlin, S.I., Inverse scaling functions in nanoindentation with sharp indenters: Determination of material properties, J. Mater. Res., 20 (2005) pp. 987-1001.

(11) Cao, Y.P. and Huber, N., Further investigation on the definition of the representative strain in conical indentation, J. Mater. Res., 21 (2006) pp. 1810-1821.

(12) Ogasawara, N., Chiba, N., Zhao, M., and Chen, X., Comments on "Further investigation on the definition of the representative strain in conical indentation" by Y. Cao and $\mathrm{N}$. Huber [J. Mater. Res. 21, 1810, (2006)], Journal of Materials Research, 22 (2007) pp. 
858-868.

(13) Ogasawara, N., Makiguchi, W., and Chiba, N., Plastic properties determination method for power-law hardening material using plural triangular pyramid indenters, Transactions of the Japan Society of Mechanical Engineers, 70 (2004) pp. 1529-1534.

(14) Ogasawara, N., Chiba, N., and Chen, X., Limit analysis-based approach to determine the material plastic properties with conical indentation, Journal of Materials Research, 21 (2006) pp. 947-958.

(15) Ogasawara, N., Chiba, N., Zhao, M., and Chen, X., Determine material plastic properties with conical indentation: an optimized framework of the representative strain, Journal of Solid Mechanics and Materials Engineering, 1 (2007) pp. 895-906.

(16) Cao, Y.P. and Lu, J., Depth-sensing instrumented indentation with dual sharp indenters: stability analysis and corresponding regularization schemes, Acta Mater., 52 (2004) pp. 1143-1153.

(17) Shu, S., Lu, J., and Li, D., A systematic study of the validation of Oliver and Pharr's method, Journal of Materials Research, 22 (2007) pp. 3385-3396.

(18) Lan, H. and Venkatesh, T.A., Determination of the elastic and plastic properties of materials through instrumented indentation with reduced sensitivity, Acta Mater., 55 (2007) pp. 2025-2041.

(19) ANSYS, Ansys Release 8.0 Documentation. 2003, Canonsburg, PA: ANSYS Inc.

(20) ABAQUS, I., ABAQUS Theory Manual. 1998, Hibbit, Karlsson and Sorensen, Inc.: Pawtucket, RI, USA.

(21) Bowden, F.P. and Tabor, D., The friction and lubrications of solids. 1950, Oxford: Oxford University Press.

(22) Hay, J.C., Bolshakov, A. and Pharr, G.M., A Critical examination of the fundamental relations used in the analysis of nanoindentation data, J. Mater. Res., 14(1999)pp.2296-2305.

(23) Alkorta, J., Martinez-Esnaola, J.M., and Gil Sevillano, J., Absence of one-to-one correspondence between elastoplastic properties and sharp-indentation load-penetration data, J. Mater. Res., 20 (2005) pp. 432-437.

(24) Oliver, W.C. and Pharr, G..M., An Improved technique for determining hardness and elastic- modulus using load and displacement sensing indentation experiments, J. Mater. Res., 7 (1992) pp. 1564-1583. 